\title{
ANÁLISIS DEL IMPACTO ECONÓMICO DE UN EVENTO DEPORTIVO. LOS JUEGOS UNIVERSITARIOS REGIONALES NEA. AÑO 2019
}

\section{ANALYSIS OF THE ECONOMIC IMPACT OF A SPORTING EVENT. NEA REGIONAL COLLEGE GAMES. EDITION 2019}

\author{
Eva Muguerza \\ Observatorio Económico y Social \\ del Deporte y la Actividad Física. \\ Universidad Nacional de Misiones \\ Posadas, Misiones, Argentina \\ evi.muguerza@gmail.com

\section{Tania Elizabet Ruff} \\ Observatorio Económico y Social \\ del Deporte y la Actividad Física. \\ Universidad Nacional de Misiones \\ Posadas, Misiones, Argentina \\ taniaruff 08@hotmail.com
}

\section{Natalia Ojeda}

Observatorio Económico y Social del Deporte y la Actividad Física. Universidad Nacional de Misiones Posadas, Misiones, Argentina nataliaojeda86@gmail.com

\author{
Francisco Mauricio Rosenfeld y Sommer \\ Observatorio Económico y Social \\ del Deporte y la Actividad Física. \\ Universidad Nacional de Misiones \\ Posadas, Misiones, Argentina \\ mauricio.rosenfeld@gmail.com
}

Fecha de recepción: 23/09/2020 - Fecha de revisión: 08/01/2021 - Fecha de aprobación: 11/01/2021

DOI: https://doi.org/10.36995/j.visiondefuturo.2021.25.01.006.es

\section{RESUMEN}

El presente trabajo constituye una contribución al campo de la economía del deporte, planteándose como objetivo principal la aplicación del modelo input output a la evaluación de impacto económico de un evento deportivo, tomando como caso de estudio los "Juegos Universitarios Regionales NEA 2019". Adicionalmente, se propone estudiar las diferentes dimensiones del legado del evento, asociado directamente al concepto de impacto.

Así este modelo permite evaluar el impacto que tiene una determinada política pública o demanda privada para estimular la actividad económica a través de un sector específico, el deporte en este caso. Para ello, se utilizan los multiplicadores de la Matriz Insumo Producto Argentina y encuestas para relevar consumos de los organizadores y participantes del evento. Todo ello, con la intención de sumar criterios de racionalidad a las decisiones que toman organizaciones deportivas tanto públicas como privadas.

Es importante mencionar que este estudio se suma a las líneas de investigación ampliamente estudiadas por Salgado Barandela, Barajas y Sánchez Fernández (2017) sobre el tema desarrolladas principalmente en Europa y Estados Unidos, no habiendo encontrado referencias académicas en Argentina, ni Latinoamérica sobre el tema.

Los resultados indican que estos juegos han generado un impulso adicional a la demanda de bienes y servicios de la ciudad sede y se ha verificado la generación de legado tanto económico como social.

\footnotetext{
“Visión de Futuro" Año 18, Volumen N$^{0} 25$ N 1, Enero - Junio 2021 - Pág 227-250 URL de la Revista: http://visiondefuturo.fce.unam.edu.ar/index.php/visiondefuturo/index URL del Documento: https://visiondefuturo.fce.unam.edu.ar/index.php/visiondefuturo/issue/view/19 ISSN 1668 - 8708 - Versión en Línea 
PALABRAS CLAVE: Impacto Económico; Evento Deportivo; Juegos Universitarios; Multiplicadores; Matriz Insumo - Producto.

\section{ABSTRACT}

The present work constitutes a contribution to the field of sports economics, considering as its main objective the application of the input output model to the evaluation of the economic impact of a sporting event, taking the "NEA 2019 Regional University Games" as a case study. Additionally, it is proposed to study the different dimensions of the event's legacy, directly associated with the concept of impact.

Thus, this model makes it possible to evaluate the impact of a specific public policy or private demand to stimulate economic activity through a specific sector, sport in this case. To do this, the multipliers of the Input Product Matrix calculated for Argentina 2004 and the consumption of the organizers and participants of the event are used. All this, with the intention of adding rationality criteria to the decisions made by both public and private sports organizations.

It is important to mention that this study joins the lines of research widely studied by Salgado Barandela, Barajas and Sánchez Fernández (2017) on the subject developed mainly in Europe and the United States, not having found academic references in Argentina or Latin America on the subject.

The results indicate that these games have generated an additional boost to the demand for goods and services of the host city and the generation of both economic and social legacy has been verified.

KEY WORDS: Economic impact; Sports Event; University Games; Multipliers; Input - Output Analysis.

\section{INTRODUCCIÓN}

\subsection{La medición de impacto económico}

En la literatura de la economía del deporte se trata la importancia del deporte en la economía en términos de su registro en las cuentas nacionales y se mide el peso del sector a partir de su participación en el PBI. Bosch, Murillo y Raya (2018) desarrollan el marco teórico y los problemas de medición que surgen al cuantificar la importancia económica del sector deportivo y el impacto económico de los eventos deportivos.

\footnotetext{
"Visión de Futuro" Año 18, Volumen N²5 N 1, Enero - Junio 2021 - Pág 227-250

URL de la Revista: http://visiondefuturo.fce.unam.edu.ar/index.php/visiondefuturo/index

URL del Documento: https://visiondefuturo.fce.unam.edu.ar/index.php/visiondefuturo/issue/view/19

ISSN 1668 - 8708 - Versión en Línea

E-mail: revistacientifica@fce.unam.edu.ar
} 
En este sentido, corresponde hacer una primera distinción entre estas dos ideas. "El peso del deporte entendido como un sector económico,(...) se puede explicar cómo el valor económico del volumen de actividades que, de manera directa o indirecta, están ligadas a las actividades deportivas" Bosch, Murillo y Raya (2018). Desde este enfoque se busca medir tanto la oferta como la demanda de deporte a nivel agregado, estudiando la producción, valor agregado, ocupación, gasto, etc.

Por su parte, "el impacto económico del deporte es un concepto más amplio y tiene que ver con los efectos de expansión que este sector promueve dentro del tejido económico global” (Bosch, García y Murillo, 2018). Por ello, se utiliza para para la medición de los efectos que tienen en la economía local la organización de eventos deportivos en diferentes escalas, tanto macro-eventos como puede ser la organización de un Juego Olímpico como de pequeña envergadura como el caso de estudio de los Juegos Universitarios Regionales NEA, 2019 que en adelante llamaremos Juegos.

Ahora bien, para cuantificar este impacto económico es necesario contar con un modelo que refleje las interrelaciones entre los diferentes sectores que participan en la cadena de valor del sector y para ello se utilizan las tablas input-output o Matriz Insumo-Producto, que tiene la capacidad para medir el efecto de la interdependencia productiva entre sectores y calcular el multiplicador que se produce cuando la economía ha recibido un shock (impulso o caída) en la demanda de algún sector (deportivo) y esto se traduce en un ajuste de todos los sectores relacionados dentro de la economía.

Aparece así el concepto de multiplicador que parte de la diferenciación entre el efecto inicial del shock y el efecto total o final que termina generándose en toda la economía por la interrelación de todos los sectores como consecuencia del efecto inicial. Es decir, que el efecto inicial en un determinado sector se ve amplificado por el flujo comercial interno de todos los sectores que, directa e indirectamente, se ven afectados. Para el desarrollo del presente estudio, se han utilizado los multiplicadores calculados por Beyrne, G. (2015) en su publicación "Análisis de Encadenamietos Productivos y Multiplicadores a partir de la construcción de la Matriz de Insumo - Producto Argentina 2004".

Asociado al concepto de impacto económico aparece la idea de legado, que tiene sentido cuando se analizan los efectos en el tiempo de la organización de un evento deportivo. Si bien esta idea de legado nace con el análisis de los Juegos Olímpicos y todas las discusiones asociadas a la gestión de inversiones y su utilización o amortización posterior a la realización de los juegos, es un concepto perfectamente aplicable y medible en eventos de menor magnitud como lo es el caso de estudio que se presenta en esta oportunidad.

\footnotetext{
"Visión de Futuro" Año 18, Volumen N$^{0} 25$ N 1, Enero - Junio 2021 - Pág 227-250

URL de la Revista: http://visiondefuturo.fce.unam.edu.ar/index.php/visiondefuturo/index

URL del Documento: https://visiondefuturo.fce.unam.edu.ar/index.php/visiondefuturo/issue/view/19

ISSN 1668 - 8708 - Versión en Línea

E-mail: revistacientifica@fce.unam.edu.ar
} 
Cuando se observa el impacto económico en el tiempo, se puede ver que existe un primer momento en el que se realiza la planificación tanto del evento como del legado y la gestión de las inversiones, que serán los primeros consumos que se llevarán a cabo para darle forma a los Juegos. El concepto de planifiación está definido en el Manual de Administración Deportiva (2014) elaborado por el Comité Olímpico Argentino (COA) como “(...) una hoja de ruta que nos guía para alcanzar nuestro destino. Tiene un propósito, una dirección y un programa: por qué, dónde y cuándo. Además, debe cubrir lo qué se debe hacer, quién debe hacerlo y, al unir los valores con las perspectivas, cómo hacerlo" Franck Dick, ex entrenador del equipo nacional británico.

Posterior a esta primera etapa, llega el momento del lanzamiento y disfrute del evento deportivo donde se realizan las actividades deportivas (juegos o competencias) planteadas en un fixture, la repercusión mediática a partir de la participación de medios de comunicación y/o la utilización de redes sociales y los consumos de los espectadores que pueden ser locales y visitantes.

Finalmente, al culminar el evento deportivo lo que queda es el legado, cuya conceptualización se desarrolla en la siguiente sección. Para representar la relación entre impacto y legado se presenta la Figura №1 con la estructura temporal mencionada anteriormente.

\begin{tabular}{|l|l|l|}
\hline Antes & Durante & Después \\
\hline Planificación & Fixture & Legados \\
Gestión de las inversiones & $\begin{array}{l}\text { Consumos de los visitantes } \\
\text { Repercusión mediática }\end{array}$ & \\
\hline
\end{tabular}

Efecto inicial de los $\begin{aligned} & \text { Efecto final de los } \\ & \text { consumos } \\ & \text { consumos }\end{aligned}$
consumos

Figura $\mathrm{N}^{\circ}$ 1: Impacto económico de un evento deportivo

Fuente: Elaboración propia

De esta manera se ha realizado un análisis temporal de impacto de eventos deportivos, segmentándolo en tres etapas: antes del evento, cuando sucede el efecto inicial; durante y después del evento, cuando se puede medir el efecto final y el legado.

\footnotetext{
"Visión de Futuro" Año 18, Volumen $N^{0} 25$ N 1, Enero - Junio 2021 - Pág 227-250

URL de la Revista: http://visiondefuturo.fce.unam.edu.ar/index.php/visiondefuturo/index

URL del Documento: https://visiondefuturo.fce.unam.edu.ar/index.php/visiondefuturo/issue/view/19

ISSN 1668 - 8708 - Versión en Línea

E-mail: revistacientifica@fce.unam.edu.ar
} 


\subsection{Impacto y legado de los eventos deportivos: el concepto de legado}

Existe multiplicidad de interpretaciones acerca del significado del "legado" del deporte y de los eventos deportivos, para elegir la definición más adecuada al momento de reflejar esta dimensión del deporte se han agrupado, seleccionado y medido cinco tipos de legados siguiendo a Walters (2013) en legado económico y social.

El legado económico es el desarrollo de capital físico y negocios y se determina a partir de los consumos e inversiones que ha provocado el evento en términos de organización y puesta en marcha, adicionando los consumos estimados de visitantes y espectadores. En este punto se puede calcular: Impacto económico y multiplicadores; Impacto en el sector hotelero; Impacto en el sector comercial; Impacto en el sector turismo; Impacto en las cuentas del Estado Nacional, Provincial y Municipal; Desarrollo de nueva infraestructura, generación de capital físico y Adaptación de infraestructura existente, puesta en valor del capital físico. El desarrollo del turismo, negocios y el potencial establecimiento de nuevas industrias en la ciudad anfitriona son algunos de los efectos que pueden suceder a la celebración de eventos deportivos.

Por su parte Hughes, Kirk y Long (2010) investigan el concepto de "legado social" en macro eventos deportivos aplicados a los Juegos Olímpicos y Paralímpicos de Londres 2012, y afirman que no están muy desarrollados los estudios empíricos sobre identificación y evaluación de la amplia gama de aspectos del legado social, que incluye: calidad de vida, cohesión comunitaria, educación, ejercicios escolares, descubrimiento de talentos, oportunidad de empleo, puesta en valor de la infraestructura, incentivos para que la población practique deporte.

Siguiendo la línea planteada por Hughes, Kirk y Long (2010) este legado se divide en:

- Legado en educación: tiene que ver con los recursos educativos, la formación de empleados y voluntarios, la investigación y la preparación antes de los eventos.

- Legado en capital humano: consiste en la formación de los implicados en la planificación y puesta en marcha de un evento.

- Legado capital social: hace referencia a las infraestructuras sociales basadas en normas sociales y de grupos, que permiten a la población de la confianza y la cooperación mutuas y por medio de las cuales, tanto los grupos como los individuos obtienen cierto tipo de ventajas Coalter (2007).

- Legado deportivo: es el legado más evidente de un evento, incluye el incentivo a la actividad física, la salud, las prácticas y hábitos deportivos que se generan a partir de la

\footnotetext{
"Visión de Futuro" Año 18, Volumen N $^{0} 5$ N 1, Enero - Junio 2021 - Pág 227-250

URL de la Revista: http://visiondefuturo.fce.unam.edu.ar/index.php/visiondefuturo/index

URL del Documento: https://visiondefuturo.fce.unam.edu.ar/index.php/visiondefuturo/issue/view/19

ISSN 1668 - 8708 - Versión en Línea

E-mail: revistacientifica@fce.unam.edu.ar
} 
influencia del evento. En este punto incorporamos la práctica deportiva con perspectiva de género.

Para conseguir que el evento sea relevante más allá de su realización, el legado de un evento debe ser un componente clave en la planificación por lo que se debe empezar a trabajar en construirlo desde antes de su inicio. Se deduce así que el legado constituye una herramienta para crear una ventaja competitiva del evento, ofreciendo un valor añadido a todo el entorno y situándolo incluso por encima del interés particular de cada agente implicado en la organización del evento deportivo.

Para el caso de los Juegos Olímpicos en Barcelona 1992, Brunet (2016) afirma que el legado se sintetiza tanto en la transformación urbana de la ciudad y en los cambios en su estructura económica como en su mayor capitalización, tercerización, internacionalización, atractivo, centralidad, productividad y competitividad. Barcelona ha logrado potenciar y mantener el impulso olímpico, incrementar su actividad y su renta, su calidad de vida y su cohesión social, y avanzar en su posición estratégica.

"De esta manera las inversiones, generan un impacto que se convierte en el núcleo del legado y en una economía global en la que la innovación y el conocimiento son la clave de la productividad, la preparación y celebración de un evento complejo con proyección mediática impulsan la competitividad. De este modo, la organización y celebración de un megaevento favorecen de un modo extraordinario la economía y la sociedad creativas". Brunet (2016).

En el desarrollo del estudio se abordarán los objetivos planteados, es decir, calcular los indicadores que permiten cuantificar el flujo económico generado por la realización de los Juegos Universitarios Regionales, NEA 2019. En adelante nos referiremos al caso de estudio como los Juegos. Este flujo se traduce en una mayor demanda de bienes y servicios locales, generación de empleo y recaudación fiscal. Asimismo, el segundo objetivo es la exploración de los legados tanto económicos (impacto final de los consumos en la economía local) como sociales de los Juegos.

\section{DESARROLLO}

\subsection{Metodología}

Con el fin de estimar el impacto económico de los Juegos, se utilizaron los multiplicadores de producción para distintas ramas de actividad calculados a partir de la Matriz Insumo Producto Argentina con datos del año 2004. Dicho método es una herramienta útil para la evaluación de impactos causados por shocks exógenos en la demanda final de un sector. De esta manera se puede calcular el impacto económico, en este caso de un evento deportivo, teniendo en cuenta "la interdependecia económica que existe entre aquellas

\footnotetext{
"Visión de Futuro" Año 18, Volumen No 25 N 1, Enero - Junio 2021 - Pág 227-250

URL de la Revista: http://visiondefuturo.fce.unam.edu.ar/index.php/visiondefuturo/index

URL del Documento: https://visiondefuturo.fce.unam.edu.ar/index.php/visiondefuturo/issue/view/19

ISSN 1668 - 8708 - Versión en Línea

E-mail: revistacientifica@fce.unam.edu.ar
} 
actividades económicas que guardan entre sí una relación recíproca". Cortés Fregoso y Sepúlveda Nuñez, (2006).

El Modelo de Insumo Producto (MIP) se compone de tres tablas básicas:

- Tabla de transacciones intersectoriales.

- Matriz de Coeficientes de Requerimientos Técnicos (o de coeficientes técnicos): se calcula a partir de la tabla de transacciones intersectoriales y expresa los requerimientos directos de insumos o valor agregado del sector que figura en la parte superior de la columna. Esto permite medir el incremento en la producción ante un aumento en la demanda final de un solo sector.

- Matriz de coeficientes de requerimientos directos e indirectos: a partir de dichos coeficientes es posible estimar el incremento de la producción total que se genera a partir del incremento de la demanda final, pero esta vez considerando el efecto en todos los sectores relacionados. Sobre esta matriz se calculan los multiplicadores de la producción descomponiéndolos en su efecto directo y su efecto indirecto.

En la estimación del impacto económico de los Juegos, se han utilizado los multiplicadores de producción para diferentes ramas de actividad calculados para la matriz de Insumo producto Argentina con datos del año 2004.

El procedimiento secuencial, ha sido el siguiente:

- A partir de la información que brindaron los organizadores, identificar y clasificar los consumos realizados para la puesta en marcha del evento deportivo

- Realizar la multiplicación de esos consumos por los multiplicadores de producción del modelo abierto de insumo-productos según rama de actividad 2004. Beyrne (2015)

- A partir de un instrumento de recolección de datos, identificar y clasificar la intención de gasto de los participantes del evento deportivo

- Realizar la multiplicación de esos consumos por los multiplicadores de producción del modelo abierto de insumo-productos según rama de actividad 2004. Beyrne (2015)

- Relacionar el consumo inicial de los organizadores con los efectos finales de esos consumos más los efectos finales de los consumos de los participantes del evento.

Por su parte, se ha diseñado un instrumento para la recolección de los datos asociados a los participantes del evento, con el propósito de contar con información pertinente con el fin de poder calcular la intención de gasto y caracterizar a los participantes (jugadores) bajo análisis y estudiar cuestiones específicas como ser la intensión del gasto de los jugadores, hábitos deportivos y motivaciones, entre otros. Para esta segunda tarea se desarrolló una encuesta que relevó diferentes dimensiones asociadas al evento:

- Intención de gasto en diferentes categorías

\footnotetext{
"Visión de Futuro" Año 18, Volumen No 25 N 1, Enero - Junio 2021 - Pág 227-250

URL de la Revista: http://visiondefuturo.fce.unam.edu.ar/index.php/visiondefuturo/index

URL del Documento: https://visiondefuturo.fce.unam.edu.ar/index.php/visiondefuturo/issue/view/19

ISSN 1668 - 8708 - Versión en Línea

E-mail: revistacientifica@fce.unam.edu.ar
} 
- Interés turístico

- Información socio ambiental de los jugadores (edad, genero, carrera, institución)

- Hábitos deportivos (práctica del deporte en la infancia y adolescencia)

- Federaciones (jugadores federados)

Para llevar a cabo dicha encuesta, se ha seleccionado una muestra a partir del método de Muestreo Aleatorio Simple (donde cada unidad de la población tiene la misma probabilidad de selección), de modo que sea posible llegar a conocer ciertas características de la población bajo análisis (todos los jugadores participantes de los Juegos) con el menor coste posible en dinero, tiempo y trabajo. López Roldán (2015).

Con el objetivo de que la muestra sea representativa de la población y de que el análisis estadístico realizado sea confiable, se han tenido en cuenta las siguientes condiciones que, según López Roldán (2015) es necesario que se cumpla a la hora de seleccionar una muestra, aunque cabe aclarar que el análisis realizado en el presente estudio es puramente descriptivo, sin llegar a realizar inferencia estadística:

- Que la muestra comprenda parte del universo y no su totalidad,

- Que el tamaño de la muestra sea estadísticamente proporcional al tamaño del universo,

- Que no existan distorsiones en el proceso de selección,

- Que sea posible poner a prueba de hipótesis sustantivas de relaciones entre variables.

El tamaño de la muestra es la cantidad de respuestas completas que la encuesta recibe y se le llama muestra porque solo representa parte del grupo de personas o población, cuyas opiniones o comportamiento son analizados para evaluar el impacto del evento. En este caso se realizó una muestra aleatoria, enviando el formulario por WhatsApp a todos los estudiantes que participaron de los Juegos. De esta manera se obtuvieron respuestas al azar de entre la población total del grupo objetivo.

La población bajo estudio estaba constituida por los jugadores que participaron en los Juegos y sumaba 1.085 estudiantes. El nivel de confianza se determinó en $99 \%$ y el margen de error en 5\%. El tamaño de la muestra resultante con estos parámetros es de 413 respuestas a las encuestas y del relevamiento realizado obtuvimos respuestas de 501 estudiantes.

La fórmula de cálculo utilizada es: $\mathrm{n}=\frac{\mathrm{z}^{2} \times \mathrm{P} \times \mathrm{Q} \times \mathrm{N}}{(\mathrm{N}-1) \times \mathrm{e}^{2}+\mathrm{z}^{2} \times \mathrm{P} \times \mathrm{Q}}$ teniendo en cuenta que se estimó el tamaño muestral para una población finita y donde se estudia una proporción.

Dónde:

$\mathrm{n}=$ tamaño de la muestra; 
$\mathrm{z}^{2}=$ el número de desvíos estándar que indica el nivel de confianza adoptado, elevado al cuadrado;

$\mathrm{e}^{2}=$ el error muestral considerado, elevado al cuadrado;

$\mathrm{N}=$ el tamaño de la población;

$\mathrm{P}=$ la proporción (o porcentaje) de individuos que tienen una característica;

$\mathrm{Q}=$ la proporción (o porcentaje) de individuos que no tienen una característica.

\subsection{Resultados}

Los Juegos Universitarios Regionales buscan promover el desarrollo académico y humano de los estudiantes universitarios con un torneo, federal, competitivo, inclusivo, innovador y solidario. Se iniciaron en 2014 y llevan 6 años realizándose en todo el país con la participación cada vez más comprometida de las instituciones que participan en la organización y gestión de los torneos.

Participan las siguientes disciplinas: ajedrez, atletismo masculino y femenino, básquetbol masculino, futsal masculino y femenino, fútbol 11 masculino y femenino, handball masculino y femenino, hockey femenino, natación masculino y femenino, rugby masculino (seven), tenis masculino y femenino, tenis de mesa, voleibol masculino y femenino. $Y$ como disciplinas promocionales beach voley, basquetbol $3 \times 3$, canotaje, ciclismo, esgrima, tiro con arco, levantamiento de pesas, judo, remo, taekwondo, tiro deportivo y yachting.

Estos Juegos Universitarios Regionales se realizan en 8 regiones, a saber: Metropolitana Sur, NOA, Bonaerense, Metropolitana Norte, Cuyo, NEA, Metropolitana CABA y Patagonia, durante un año y de cada región los campeones en cada categoría juegan los JUR nacionales al siguiente año. En el caso de los JURNEA han participado 11 instituciones (públicas y privadas) de Formosa, Chaco, Corrientes y Misiones durante 4 días donde compitieron alrededor de 1.100 estudiantes.

\subsubsection{Antes del evento}

La organización y planificación de los Juegos estuvo a cargo de la sede, en este caso la Universidad Nacional de Misiones con el apoyo de la Federación del Deporte Universitario.

Como parte de la planificación del legado, el Observatorio Económico y Social del deporte y la actividad física de la UNaM trabajo en la identificación y valoración del impacto económico y legado. Para ello se avanzó en dos etapas, una inicial con el detalle de los egresos y actividades que conlleva la organización del evento y una segunda etapa, en la que

\footnotetext{
"Visión de Futuro" Año 18, Volumen No 25 N 1, Enero - Junio 2021 - Pág 227-250

URL de la Revista: http://visiondefuturo.fce.unam.edu.ar/index.php/visiondefuturo/index

URL del Documento: https://visiondefuturo.fce.unam.edu.ar/index.php/visiondefuturo/issue/view/19

ISSN 1668 - 8708 - Versión en Línea

E-mail: revistacientifica@fce.unam.edu.ar
} 
se apoyó en las instituciones locales e invitadas para cuantificar los gastos esperados producto del evento.

Los ingresos con los que habitualmente se financian los eventos deportivos vienen de fuentes públicas y privadas, a saber: Estado nacional, Estado provincial, Estado municipal, Empresas patrocinadoras, Derechos de TV, Entradas y Donaciones. Estos ingresos pueden darse en moneda o en especies como acceso a instalaciones deportivas, seguridad, salud, indumentaria, material deportivo, medallas, etc.

En relación a la gestión de las compras y las inversiones se pudo verificar que los ingresos principales que han permitido financiar los Juegos tienen su origen en el Presupuesto de la Nación, dentro del Ministerio de Educación. Adicionalmente, el gobierno de la Provincia colaboro con recursos monetarios y en especies (instalaciones deportivas y alojamiento). En esta ocasión no hubo ingresos por entradas, derechos televisivos, ni patrocinadores privados. La administración de los recursos estuvo a cargo de la UNaM, Secretaría General de Extensión - Asuntos Estudiantiles.

Por otra parte, los egresos y consumos generados antes y durante el evento se realizaron principalmente en la Ciudad de Posadas. La información referente a los consumos iniciales asociados a la organización del evento deportivo, se presentan en la Tabla 1.

Tabla №1: Consumo generado en la organización del evento en pesos corrientes y en porcentajes

\begin{tabular}{|r|l|c|c|}
\hline Item & \multicolumn{1}{|c|}{ Concepto } & Monto en \$ & $\%$ sobre el total \\
\hline 1 & Alimentos & $\$ 438.110,00$ & $11 \%$ \\
\hline 5 & Productos textiles & $\$ 170.850,00$ & $4 \%$ \\
\hline 7 & Transporte aereo, cargas y pasajeros & $\$ 1.289 .790,00$ & $33 \%$ \\
\hline 11 & Ss. De hotelería y restaurantes & $\$ 1.172 .700,00$ & $30 \%$ \\
\hline 13 & Cinematografía, radio y TV & $\$$ & - \\
\hline 15 & Ss. De organizaciones empresariales & $\$ 605.319,00$ & $15 \%$ \\
\hline 21 & Seguros y AFIP & $\$ 69.454,00$ & $2 \%$ \\
\hline 51 & Comercio por menor & $\$ 197.957,00$ & $5 \%$ \\
\hline & Totales & $\mathbf{\$ 3 . 9 4 4 . 1 8 0 , 0 0}$ & $\mathbf{1 0 0 \%}$ \\
\hline
\end{tabular}

Fuente: Elaboración Propia

\subsubsection{Durante el evento}

\section{A. Fixture}

Los organizadores del evento realizan el fixture, una vez que se han cerrado las acreditaciones y en función de los deportistas que se presentaron para cada deporte de grupo o individual. El desarrollo de los juegos puede realizarse en una sede o mas según sea la

\footnotetext{
"Visión de Futuro" Año 18, Volumen No 25 N 1, Enero - Junio 2021 - Pág 227-250

URL de la Revista: http://visiondefuturo.fce.unam.edu.ar/index.php/visiondefuturo/index

URL del Documento: https://visiondefuturo.fce.unam.edu.ar/index.php/visiondefuturo/issue/view/19

ISSN 1668 - 8708 - Versión en Línea

E-mail: revistacientifica@fce.unam.edu.ar
} 
disponibilidad de la ciudad de infraestructura adecuada a los deportes en competición. Como resultado del fixture y el rendimiento de los equipos se presenta la Tabla №2 donde se resume la información de los tres primeros puestos por deporte y por categoría.

Tabla №2: Resultados por deporte y categorías

\begin{tabular}{|c|c|c|c|c|c|c|}
\hline \multirow{2}{*}{ Resultados/ Deportes } & \multicolumn{3}{|c|}{ Mujeres } & \multicolumn{3}{|c|}{ Varones } \\
\hline & $1^{\circ}$ & $2^{\circ}$ & $3^{\circ}$ & $1^{\circ}$ & $2^{\circ}$ & $3^{\circ}$ \\
\hline Ajedrez & $\begin{array}{c}\text { Sin } \\
\text { participantes }\end{array}$ & $\begin{array}{c}\text { Sin } \\
\text { participantes }\end{array}$ & $\begin{array}{c}\text { Sin } \\
\text { participantes }\end{array}$ & $\begin{array}{c}\text { Universidad } \\
\text { Nacional de } \\
\text { Formosa }\end{array}$ & $\begin{array}{c}\text { Universidad } \\
\text { Nacional de } \\
\text { Misiones }\end{array}$ & $\begin{array}{c}\text { Universidad } \\
\text { Nacional de } \\
\text { Chaco Austral }\end{array}$ \\
\hline Atletismo & $\begin{array}{c}\text { IESEF } \\
\text { Resistencia }\end{array}$ & $\begin{array}{c}\text { ISEF Antonio } \\
\text { Alejandro } \\
\text { Alvarez } \\
\end{array}$ & $\begin{array}{c}\text { Universidad } \\
\text { Nacional del } \\
\text { Nordeste } \\
\end{array}$ & ISEF - IESEF & - & $\begin{array}{c}\text { Universidad } \\
\text { Nacional del } \\
\text { Nordeste }\end{array}$ \\
\hline Basquet $3 \times 3$ & $\begin{array}{c}\text { Universidad } \\
\text { Nacional de } \\
\text { Misiones }\end{array}$ & $\begin{array}{c}\text { IESEF } \\
\text { Resistencia }\end{array}$ & $\begin{array}{c}\text { Barceló } \\
\text { Santo Tomé }\end{array}$ & - & - & - \\
\hline Basquetbol & - & - & - & $\begin{array}{c}\text { Universidad } \\
\text { Nacional de } \\
\text { Chaco Austral }\end{array}$ & $\begin{array}{c}\text { IESEF } \\
\text { Resistencia }\end{array}$ & $\begin{array}{l}\text { Instituto Sup } \\
\text { Antonio Ruiz } \\
\text { de Montoya }\end{array}$ \\
\hline Futbol 11 & $\begin{array}{c}\text { Universidad } \\
\text { Nacional de } \\
\text { Formosa }\end{array}$ & $\begin{array}{l}\text { Universidad } \\
\text { Nacional de } \\
\text { Misiones } \\
\end{array}$ & & \begin{tabular}{|c|}
$\begin{array}{c}\text { Universidad } \\
\text { Nacional del } \\
\text { Nordeste }\end{array}$ \\
\end{tabular} & $\begin{array}{c}\text { Universidad } \\
\text { Nacional de } \\
\text { Misiones } \\
\end{array}$ & $\begin{array}{c}\text { IESEF } \\
\text { Resistencia }\end{array}$ \\
\hline Futsal & $\begin{array}{c}\text { IESEF } \\
\text { Resistencia }\end{array}$ & $\begin{array}{l}\text { Universidad } \\
\text { Nacional de } \\
\text { Misiones } \\
\end{array}$ & $\begin{array}{c}\text { Universidad } \\
\text { Nacional del } \\
\text { Nordeste } \\
\end{array}$ & $\begin{array}{c}\text { Universidad } \\
\text { Nacional de } \\
\text { Misiones } \\
\end{array}$ & \begin{tabular}{|c|} 
Universidad \\
Nacional del \\
Nordeste
\end{tabular} & \begin{tabular}{|c|} 
ISEF Antonio \\
Alejandro \\
Alvarez
\end{tabular} \\
\hline Handball & $\begin{array}{c}\text { Universidad } \\
\text { Nacional de } \\
\text { Formosa }\end{array}$ & $\begin{array}{c}\text { IESEF } \\
\text { Resistencia }\end{array}$ & $\begin{array}{l}\text { Universidad } \\
\text { Nacional de } \\
\text { Misiones }\end{array}$ & $\begin{array}{c}\text { Universidad } \\
\text { Nacional del } \\
\text { Nordeste }\end{array}$ & $\begin{array}{c}\text { Universidad } \\
\text { Nacional de } \\
\text { Chaco Austral }\end{array}$ & $\begin{array}{l}\text { ISEF Antonio } \\
\text { Alejandro } \\
\text { Alvarez }\end{array}$ \\
\hline Hockey & $\begin{array}{c}\text { Universidad } \\
\text { Nacional de } \\
\text { Misiones }\end{array}$ & $\begin{array}{c}\text { ISEF Antonio } \\
\text { Alejandro } \\
\text { Alvarez }\end{array}$ & $\begin{array}{c}\text { Universidad } \\
\text { Nacional del } \\
\text { Nordeste } \\
\end{array}$ & \begin{tabular}{|} 
Sin \\
participantes
\end{tabular} & $\underset{\text { participantes }}{\mathrm{Sin}}$ & $\begin{array}{c}\text { Sin } \\
\text { participantes }\end{array}$ \\
\hline Natación & $\begin{array}{l}\text { Instituto Sup } \\
\text { Antonio Ruiz } \\
\text { de Montoya }\end{array}$ & $\begin{array}{c}\text { IESEF } \\
\text { Resistencia }\end{array}$ & $\begin{array}{c}\text { ISEF Antonio } \\
\text { Alejandro } \\
\text { Alvarez } \\
\end{array}$ & $\begin{array}{c}\text { Universidad } \\
\text { Nacional de } \\
\text { Misiones } \\
\end{array}$ & $\begin{array}{c}\text { IESEF } \\
\text { Resistencia }\end{array}$ & $\begin{array}{c}\text { ISEF Antonio } \\
\text { Alejandro } \\
\text { Alvarez } \\
\end{array}$ \\
\hline Rugby 7 & $\underset{\text { participantes }}{\text { Sin }}$ & $\underset{\text { participantes }}{\text { Sin }}$ & $\begin{array}{c}\text { Sin } \\
\text { participantes }\end{array}$ & \begin{tabular}{|c|} 
ISEF Antonio \\
Alejandro \\
Alvarez
\end{tabular} & $\begin{array}{c}\text { IESEF } \\
\text { Resistencia }\end{array}$ & $\begin{array}{l}\text { Universidad } \\
\text { Nacional de } \\
\text { Misiones }\end{array}$ \\
\hline Tenis & $\begin{array}{c}\text { ISEF Antonio } \\
\text { Alejandro } \\
\text { Alvarez }\end{array}$ & - & - & \begin{tabular}{|c|} 
ISEF Antonio \\
Alejandro \\
Alvarez \\
\end{tabular} & $\begin{array}{c}\text { Universidad } \\
\text { Nacional de } \\
\text { Misiones } \\
\end{array}$ & - \\
\hline Tenis de mesa & $\begin{array}{c}\text { Sin } \\
\text { participantes }\end{array}$ & $\begin{array}{c}\text { Sin } \\
\text { participantes }\end{array}$ & $\begin{array}{c}\text { Sin } \\
\text { participantes }\end{array}$ & \begin{tabular}{|c|}
$\begin{array}{c}\text { Universidad } \\
\text { Nacional del } \\
\text { Nordeste }\end{array}$ \\
\end{tabular} & $\begin{array}{c}\text { Universidad } \\
\text { Nacional de } \\
\text { Misiones } \\
\end{array}$ & \begin{tabular}{|c|} 
ISEF Antonio \\
Alejandro \\
Alvarez
\end{tabular} \\
\hline Voleibol & $\begin{array}{c}\text { Universidad } \\
\text { Nacional del } \\
\text { Nordeste }\end{array}$ & $\begin{array}{c}\text { IESEF } \\
\text { Resistencia }\end{array}$ & $\begin{array}{c}\text { Universidad } \\
\text { Nacional de } \\
\text { Formosa }\end{array}$ & $\begin{array}{c}\text { IESEF } \\
\text { Resistencia }\end{array}$ & $\begin{array}{c}\text { Universidad } \\
\text { Nacional de } \\
\text { Formosa }\end{array}$ & $\begin{array}{l}\text { Universidad } \\
\text { Nacional de } \\
\text { Misiones }\end{array}$ \\
\hline
\end{tabular}

Fuente: Elaboración propia

Como puede observarse en la tabla, el top 5 está conformado por el IESEF Resistencia, que logró posicionarse en podio con 14 medallas, seguido por la UNaM con 13 medallas, en tanto el ISEF Antonio Alejandro Alvarez hizo podio en 11 competencias, seguida por la Universidad Nacional del Nordeste que logó 8 medallas y finalmente la Universidad Nacional de Formosa con 5 medallas.

\footnotetext{
"Visión de Futuro" Año 18, Volumen No 25 No 1, Enero - Junio 2021 - Pág 227-250

URL de la Revista: http://visiondefuturo.fce.unam.edu.ar/index.php/visiondefuturo/index

URL del Documento: https://visiondefuturo.fce.unam.edu.ar/index.php/visiondefuturo/issue/view/19

ISSN 1668 - 8708 - Versión en Línea

E-mail: revistacientifica@fce.unam.edu.ar
} 
Es interesante señalar que le IESEF Resistencia obtuvo el $32 \%$ de las medallas con el $12 \%$ de competidores respecto del total de jugadores de los Juegos, en tanto la UNaM logro el $30 \%$ de medallas con $20 \%$ de participantes. Por su parte, la distribución de medallas estuvo muy pareja ya que de las 11 instituciones que participaron, el $73 \%$ hizo podio con sus equipos o deportistas individuales mostrando un buen balance competitivo

\section{B. Repercusión Mediática}

Es importante identificar el grado mediático que adquiere el evento, con el fin de estimar lo ingresos potenciales o reales por derechos de TV. De ser posible, se deben identificar también las repercusiones en internet y redes sociales: Sitio web, Youtube, Facebook, Instagam, Twiter, Whats App.

Al ser un evento que involucro a institutos y universidades públicas y privadas, UNaM Transmedia decidió trabajar la difusión y la repercusión a partir de las plataformas digitales, es decir redes sociales y una página web creada especialmente para los juegos: www.jur.UNaM.edu.ar. El sitio se creó con el objetivo de ser un repositorio donde confluyeran las producciones de las redes sociales relacionadas al encuentro e información de relevancia para la misma. Se puso en línea para el público el jueves 7 de noviembre y desde ese momento hasta los primeros días de diciembre hubo 8.194 visitas, 72 comentarios. El día con más visitas fue el lunes 11 de noviembre con 4.064 visitas.

Se realizó una cobertura mediática con un formato periodístico y documental. La idea era contar "historias" es decir se desarrolló un universo narrativo a través de las redes sociales generando historias y un sitio www.jur.UNaM.edu.ar. Durante el evento se hicieron programas donde se registró lo que sucedió en los campos de juego. Se subieron los productos televisivos y documentos que cubrieron esa narrativa, que se desarrolla en plataformas mediales (radio, podcast, etc) y ese universo está abierto y permite la coautoría del espectador, generando contenido también.

Para los juegos, se logró integrar y fusionar las carreras de comunicación social de Misiones y de Corrientes, los estudiantes y profesores trabajaron a la par en el registro de todo el evento. UNaM Transmedia aseguró el equipamiento necesario para la cobertura de los juegos y se pensó en una cobertura en red y online. Se generaron piezas (videos y podcast) que serviría para múltiples propósitos. También hubo una cobertura colaborativa en Oberá con UNaM Transmedia, la carrera de fotografía y medios audiovisuales de la Facultad de Artes de la UNaM, Corrientes y Chaco.

\footnotetext{
“Visión de Futuro" Año 18, Volumen $N^{0} 25$ N$^{0}$ 1, Enero - Junio 2021 - Pág 227-250

URL de la Revista: http://visiondefuturo.fce.unam.edu.ar/index.php/visiondefuturo/index

URL del Documento: https://visiondefuturo.fce.unam.edu.ar/index.php/visiondefuturo/issue/view/19

ISSN 1668 - 8708 - Versión en Línea

E-mail: revistacientifica@fce.unam.edu.ar
} 
En las producciones participaron más de 50 personas entre estudiantes, técnicos, camarógrafos, uno de ellos del centro de producción audiovisual de Formosa, Chaco, Corrientes y Misiones. Se integraron equipos de las áreas de comunicación, audiovisual, tecnológicas hubo un grupo de informáticos haciendo el apoyo y actualización de la web. Gente con diferentes roles, tareas trabajando de manera colaborativa y nutrida.

Se realizaron producciones a modo de resumen de cada jornada con información y entrevistas que salieron en formato televisivo. Así se trabajó en múltiples áreas, múltiples formatos y múltiples pantallas.

\section{Consumos jugadores}

Los visitantes realizarán gastos durante el transcurso del tiempo que dure el evento y se podrá estimar a partir de encuestas elaboradas a tal efecto. Los ítems que habitualmente se consideran son: Alojamiento, Transporte, Alimentos, Turismo, Ocio y Tiendas.

Por las características de los Juegos en donde el alojamiento, la alimentación y los traslados estaban incluidos en la inscripción de los participantes de las universidades públicas, se decidió evaluar los gastos adicionales que realizarían los jugadores tanto locales como visitantes a partir de una tabla que los encuestados completaron con opciones de rangos monetarios y actividades en las cuales aplicaron esos recursos.

Las actividades que resultaban de interés evaluar fueron:

- Ocio que incluye las salidas nocturnas y en los ratos libres (confiterías, discotecas, etc).

- Turismo

- Tiendas de recuerdos, presentes, ropa

- Alimentos fuera del hotel

- Otros

Tabla №3: Intención de gasto por tipo de actividad para jugadores/as

\begin{tabular}{|c|c|c|c|c|c|c|c|c|c|c|}
\hline \multirow{2}{*}{\begin{tabular}{|r|} 
Intención de Gasto \\
Ocio (confiterías, discotecas)
\end{tabular}} & \multicolumn{2}{|c|}{ de $\$ 0$ a $\$ 500$} & \multicolumn{2}{|c|}{$\begin{array}{c}\text { de } \$ 501 \text { a } \\
\$ 1.000\end{array}$} & \multicolumn{2}{|c|}{$\begin{array}{c}\text { de } \$ 1.000 \text { a } \\
\$ 1.500\end{array}$} & \multicolumn{2}{|c|}{$\begin{array}{c}\text { más de } \\
\$ 1.500\end{array}$} & \multicolumn{2}{|r|}{$\begin{array}{l}\text { Consumo } \\
\text { Promedio }\end{array}$} \\
\hline & 362 & $72 \%$ & 90 & $18 \%$ & 39 & $8 \%$ & 10 & $2 \%$ & $\$$ & 443,00 \\
\hline Turismo & 328 & $65 \%$ & 117 & $23 \%$ & 44 & $9 \%$ & 12 & $2 \%$ & $\$$ & 485,00 \\
\hline Tiendas (regalos, recuerdos, ropa) & 344 & $69 \%$ & 103 & $21 \%$ & 37 & $7 \%$ & 17 & $3 \%$ & $\$$ & 469,00 \\
\hline Alimentos (fuera de los comedores) & 309 & $62 \%$ & 136 & $27 \%$ & 48 & $10 \%$ & 8 & $2 \%$ & $\$$ & 501,00 \\
\hline Otros & 399 & $80 \%$ & 66 & $13 \%$ & 24 & $50 \%$ & 12 & $2 \%$ & $\$$ & 394,00 \\
\hline \multicolumn{9}{|c|}{ Consumo estimado medio } & $\$$ & $2.291,00$ \\
\hline \multicolumn{9}{|c|}{ Total consumo estimado } & $\$$ & $2.486 .188,00$ \\
\hline
\end{tabular}

Fuente: Elaboración propia (moneda corriente 2019)

\footnotetext{
“Visión de Futuro" Año 18, Volumen N$^{0} 25$ N 1, Enero - Junio 2021 - Pág 227-250

URL de la Revista: http://visiondefuturo.fce.unam.edu.ar/index.php/visiondefuturo/index

URL del Documento: https://visiondefuturo.fce.unam.edu.ar/index.php/visiondefuturo/issue/view/19

ISSN 1668 - 8708 - Versión en Línea

E-mail: revistacientifica@fce.unam.edu.ar
} 
De acuerdo a la información presentada en la Tabla №3, los rangos hasta $\$ 1.000$ son los que tienen mayor presencia en el gasto estimado de los estudiantes. En particular el $72 \%$ de los encuestados manifestó que gastaría entre $\$ 0$ y $\$ 500$ en ocio y el $69 \%$ gastaría en tiendas de regalos y recuerdos. En turismo el $65 \%$ de los jugadores estaría interesado en gastar en ese primer rango también.

El perfil de consumo de los estudiantes genera un gasto promedio estimado en \$2.291 y cuando lo calculamos para todos los jugadores que han participado de los juegos, el monto asciende a $\$ 2.486 .188$.- que han incrementado la demanda de bienes y servicios en las localidades anfitrionas, Posadas y Oberá, siendo el mayor consumo en la Ciudad de Posadas.

\subsubsection{Después del evento}

\section{A. Legado económico: Impacto económico y multiplicadores}

Una vez identificados todos los gastos asociados a la organización del evento y los gastos estimados de los jugadores, es el momento de calcular los efectos iniciales y finales del evento, tal como se muestra en la Tabla 4.

Tabla №4: Estimación de los efectos finales por gastos de la organización y de los jugadores

\begin{tabular}{|c|c|c|c|c|c|c|c|c|c|c|c|}
\hline Item & Concepto & Inicial & Directo & Indirecto & Efecto Total & $\begin{array}{c}\text { Consumos } \\
\text { Organización }\end{array}$ & & $\begin{array}{l}\text { Consumos } \\
\text { jugadores }\end{array}$ & & $\begin{array}{l}\text { Consumos } \\
\text { Totales }\end{array}$ & Efecto Total \\
\hline 1 & Alimentos & 1 & 0,699 & 0,534 & 2,233 & $438.110,00$ & $\$$ & $1.024 .361,00$ & $\$$ & $1.462 .471,00$ & $\$ 3.265 .697,74$ \\
\hline 5 & Productos textiles & 1 & 0,525 & 0,425 & 1,95 & $170.850,00$ & $\$$ & - & $\$$ & $170.850,00$ & $333.157,50$ \\
\hline 7 & Transporte aereo, cargas y pasajeros & 1 & 0,516 & 0,413 & 1,929 & $\$ 1.289 .790,00$ & $\$$ & - & $\$$ & $1.289 .790,00$ & $\$ 2.488 .004,91$ \\
\hline 11 & Ss. De hotelería y restaurantes & 1 & 0,459 & 0,401 & 1,86 & $\$ 1.172 .700,00$ & $\$$ & - & $\$$ & $1.172 .700,00$ & $\$ 2.181 .222,00$ \\
\hline 13 & Cinematografía, radio y TV & 1 & 0,481 & 0,346 & 1,827 & $\$$ & $\$$ & - & $\$$ & - & $\$$ \\
\hline 15 & Ss. De organizaciones empresariales & 1 & 0,479 & 0,333 & 1,812 & $605.319,00$ & $\$$ & $525.716,00$ & $\$$ & $1.131 .035,00$ & $\$ 2.049 .435,42$ \\
\hline 21 & Seguros y AFIP & 1 & 0,477 & 0,305 & 1,782 & $69.454,00$ & $\$$ & - & $\$$ & $69.454,00$ & $123.767,03$ \\
\hline 51 & Comercio por menor & 1 & 0,261 & 0,155 & 1,416 & $\$ \quad 197.957,00$ & $\$$ & $936.110,00$ & $\$$ & $1.134 .067,00$ & $\$ 1.605 .838,87$ \\
\hline \multicolumn{6}{|c|}{ Totales } & $\$ 3.944 .180,00$ & $\$$ & $2.486 .187,00$ & $\$$ & $6.430 .367,00$ & $\$ 12.047 .123,47$ \\
\hline
\end{tabular}

Fuente: Elaboración propia (moneda corriente de 2019)

Así \$3.944.168 sirvieron para financiar los consumos que genero la organización del evento y que conformaron el impacto inicial, junto a $\$ 2.486 .168$ que consumieron los jugadores.

Todo esto, se tradujo en un aumento de la demanda de bienes y servicios en su mayoría locales, cuyo impacto luego de los ajustes en la cadena de valor de cada consumo fue de $\$ 12.047 .126$. Esto significa que el consumo inicial generado por los Juegos se multiplicó 3 veces cuando se desencadenó la cadena de gastos asociados al evento.

\footnotetext{
“Visión de Futuro" Año 18, Volumen $N^{0} 25$ N$^{0}$, Enero - Junio 2021 - Pág 227-250

URL de la Revista: http://visiondefuturo.fce.unam.edu.ar/index.php/visiondefuturo/index

URL del Documento: https://visiondefuturo.fce.unam.edu.ar/index.php/visiondefuturo/issue/view/19

ISSN 1668 - 8708 - Versión en Línea

E-mail: revistacientifica@fce.unam.edu.ar
} 


\section{A.1 Ingresos del Sector Público}

Cuando consideramos el consumo total realizado con motivo de los Juegos, $\$ 12.047 .126$.- podemos calcular los ingresos estimados por recaudación fiscal, en los diferentes niveles de gobierno:

- IVA (21\% sobre los consumos)

- $\operatorname{lIBB}(4,5 \%$ sobre la facturación)

- Tasas Municipales (0,01\% sobre la facturación descontando IIBB)

Los ingresos fiscales gracias a los consumos generados por el evento ascienden a \$3.073.222 de los cuales en la Provincia de Misiones se quedan \$543.326.-

\section{A.2 Desarrollo de nueva infraestructura y/o adaptación/amortización de infraestructura existente}

En estos juegos no fue necesaria la adaptación, reparación o generación de infraestructura. Se utilizó el capital físico existente.

\section{A.3 Incremento del turismo}

Dada la importancia de este sector económico, consideramos pertinente hacer un análisis complementario de los efectos del evento en la actividad hotelera. Y para ello, se han realizado entrevistas personales con la gerencia de estos establecimientos para contextualizar el sector y analizar correctamente el impacto del evento en este rubro.

En la ciudad de Posadas se han registrado para 2016, 49 establecimientos totalizando 1.315 habitaciones y 3.375 plazas según el Anuario estadístico de la Provincia de Misiones 2016 - IPEC). De estos 49 establecimientos, para el evento se han ocupado parcialmente 3 es decir el $6 \%$ de la oferta de hoteles en la ciudad.

Se realizaron entrevistas a los hoteles para estimar los ingresos y la ocupación generada por los Juegos. Los resultados se pueden visualizar en la Tabla №5 donde se han seleccionado los indicadores relevantes para medir la actividad hotelera a partir del aumento de la demanda que han generado los Juegos.

Tabla №5: Indicadores de la actividad hotelera la semana de los JURNEA2019

\begin{tabular}{|l|r|}
\hline Plazas totales ocupadas por el evento en 3 hoteles de la Ciudad de Posadas en los 4 días & 1.548 \\
\hline Nivel promedio de ocupación durante los días del evento en los 3 hoteles & $81 \%$ \\
\hline Tasa de crecimiento de la ocupación respecto del año anterior & $132 \%$ \\
\hline Ingreso generado a los hoteles, en concepto de alojamiento & $\mathbf{\$ 1 . 1 7 2 . 7 0 0 , 0 0}$ \\
\hline Ocupación de la oferta disponible de hoteles de la Ciudad de Posadas (49) & $6 \%$ \\
\hline
\end{tabular}

Fuente: Elaboración propia

\footnotetext{
"Visión de Futuro" Año 18, Volumen N²5 N 1, Enero - Junio 2021 - Pág 227-250

URL de la Revista: http://visiondefuturo.fce.unam.edu.ar/index.php/visiondefuturo/index

URL del Documento: https://visiondefuturo.fce.unam.edu.ar/index.php/visiondefuturo/issue/view/19

ISSN 1668 - 8708 - Versión en Línea

E-mail: revistacientifica@fce.unam.edu.ar
} 
Las primeras conclusiones interesantes que podemos destacar son que: a) Los Juegos se tradujeron en una ocupación de entre el $81 \%$ en los hoteles utilizados. Y ello representó ingresos solo por el evento de \$1.172.700 por alojamiento entre los tres hoteles; b) La tasa de ocupación estimada aumento en 132\% respecto del año 2018, generando ingresos adicionales para estos hoteles.

Es importante destacar que la estadía promedio de los turistas o visitantes en Posadas es de 1,7 días, según los datos que relva el IPEC en el informe mencionado anteriormente. Por lo que un evento que ocupa la capacidad hotelera por cuatro días es relevante para actividad del sector.

\section{B. Legado social: desarrollo de capital humano}

\section{B.1 Legado en educación - educación de los deportistas}

En este espacio se ha estudiado el perfil demográfico y deportivo de los jugadores. Es posible observar que la edad con mayor rango de participación (38\%) es la que va de 21 a 23 años, seguida por el rango de 18 a 20 años con un 27\%. En tercer lugar, se encuentra el rango que va desde 24 a 26 años con un $20 \%$ y por último, los atletas con 27 años o más con una participación del 15\%.

En la Tabla №6 se presenta la información relativa a la proporción de jugadores/as por carrera agrupados en las ciencias de la educación física, ingenierías, ciencias de la educación, ciencias de la salud, ciencias económicas, ciencias exactas y naturales, ciencias humanas y sociales, ciencias jurídicas, ciencias veterinarias, forestales y agrarias.

Los datos muestran que la mayor cantidad de jugadores/as (19\%) son de las ciencias de la educación física, seguidos muy cerca con un 18\% por las ingenierías, ciencias de la educación (16\%) y ciencias de la salud (14\%).

\footnotetext{
"Visión de Futuro" Año 18, Volumen N $^{0} 5$ N 1, Enero - Junio 2021 - Pág 227-250

URL de la Revista: http://visiondefuturo.fce.unam.edu.ar/index.php/visiondefuturo/index

URL del Documento: https://visiondefuturo.fce.unam.edu.ar/index.php/visiondefuturo/issue/view/19

ISSN 1668 - 8708 - Versión en Línea

E-mail: revistacientifica@fce.unam.edu.ar
} 
Tabla №6 Jugadores agrupados por carrera/ciencia de los equipos visitantes

\begin{tabular}{|l|c|c|}
\hline \multicolumn{1}{|c|}{ Carrera/Ciencia } & Cantidad & $\begin{array}{c}\% \text { de } \\
\text { participació } \\
\mathbf{n}\end{array}$ \\
\hline Educación física & 96 & $19 \%$ \\
\hline Ingenierías & 90 & $18 \%$ \\
\hline Cs. De la Educación & 78 & $16 \%$ \\
\hline Cs. De la Salud & 71 & $14 \%$ \\
\hline Cs. Económicas & 50 & $10 \%$ \\
\hline Cs. Exactas y naturales & 37 & $7 \%$ \\
\hline Cs. Humanas y sociales & 34 & $7 \%$ \\
\hline Cs. Jurídicas & 17 & $3 \%$ \\
\hline Cs. Veterinarias, forestale y agrarias & 12 & $2 \%$ \\
\hline Otras & 16 & $3 \%$ \\
\hline \multicolumn{1}{|c|}{ Totales } & $\mathbf{5 0 1}$ & $\mathbf{1 0 0 \%}$ \\
\hline
\end{tabular}

Fuente: Elaboración Propia

\section{B.2 Legado en capital humano}

Consiste en la formación de los implicados en la planificación y puesta en marcha de un evento. Se considera un legado deseable ya que genera el Know how para la organización de otros eventos en el futuro (voluntarios, gestores, redes de empresas que son funcionales al evento). Respecto al Voluntariado, durante los juegos trabajaron 15 voluntarios para acreditaciones, y en todos los deportes colaborando con los profesores, además de las mesas de control.

Además, el desarrollo del conocimiento para la organización de eventos deportivos es importante para asegurar el éxito del presente y futuros juegos. En el caso de los JUR, el equipo organizador tiene antecedentes en múltiples eventos y una de las gestiones realizadas en este caso fue la coordinación con diferentes áreas de Gobierno.

\section{B.3 Legado capital social - Sentido de pertenencia de la población}

Resulta interesante comprender las motivaciones de los estudiantes para participar de los Juegos, para ello se realizó una pregunta abierta en el cuestionario y se agruparon las 501 respuestas en función de las motivaciones más relevantes que se exponen en la Tabla №7.

\footnotetext{
“Visión de Futuro" Año 18, Volumen $N^{0} 25$ N$^{0}$ 1, Enero - Junio 2021 - Pág 227-250

URL de la Revista: http://visiondefuturo.fce.unam.edu.ar/index.php/visiondefuturo/index

URL del Documento: https://visiondefuturo.fce.unam.edu.ar/index.php/visiondefuturo/issue/view/19

ISSN 1668 - 8708 - Versión en Línea

E-mail: revistacientifica@fce.unam.edu.ar
} 
Tabla N7: Principales motivadores en los JURNEA2019

\begin{tabular}{|l|r|r|}
\hline \multicolumn{1}{|c|}{ Motivo } & \multicolumn{1}{c|}{ Cantidad } & \multicolumn{1}{c|}{$\%$} \\
\hline Pasión/ amor por el deporte y la competencia & 188 & $38 \%$ \\
\hline $\begin{array}{l}\text { Representar a la institución/equipo y acceder a los } \\
\text { nacionales }\end{array}$ & 109 & $22 \%$ \\
\hline $\begin{array}{l}\text { Experiencia en lo deportivo, cultura, turismo, } \\
\text { educación }\end{array}$ & 96 & $19 \%$ \\
\hline No responde & 65 & $13 \%$ \\
\hline $\begin{array}{l}\text { Amistad, compañerismo, convocatoria, trabajo en } \\
\text { equipo }\end{array}$ & 43 & $9 \%$ \\
\hline Total encuestas & $\mathbf{5 0 1}$ & $\mathbf{1 0 0} \%$ \\
\hline
\end{tabular}

Fuente: Elaboración propia

El principal estímulo surge del amor al deporte y a la competencia (38\%). Y la posibilidad de representar a la institución de origen y de lograr la clasificación a los juegos nacionales es la segunda motivación en orden de importancia, $22 \%$.

\section{B.4 Legado deportivo}

\section{B.4.1 Práctica deportiva en la población (hábitos deportivos)}

Bertrán (2005) realiza las siguientes consideraciones:

“...tradicionalmente, se ha visto el deporte como una actividad lúdica basada en el esfuerzo físico y la competición que distrae a los niños y adolescentes del rendimiento académico, eminentemente mental, pero también cimentado en el esfuerzo y la competencia. En el imaginario colectivo de padres, educadores, gestores sociales y políticos, la actividad formativa de los adolescentes corresponde fundamentalmente al rendimiento intelectual, que no físico".

Múltiples estudios rompen el mito sobre la relación negativa entre el rendimiento académico y la actividad física y el deporte, es más refuerzan el papel positivo que tienen las prácticas físicas sistematizadas en el crecimiento personal de los adolescentes y en su encaje social. De esta línea de pensamiento, se desprende que la actividad física y el deporte deberían ser el eje conductor de políticas públicas enfocadas en la promoción de la práctica regular de deporte en la etapa adolescente y así poder colaborar eficazmente en el crecimiento personal de los jóvenes.

Al enfocar el análisis en los jugadores/as y sus hábitos deportivos, cuya información se resume en la Tabla №8, puede observarse que del total encuestado, el 67\% asegura que ha practicado regularmente deportes entre los 13 y los 17 años; el 50\% que lo ha hecho entre los 18 y 25 años; y el 44\% en su niñez (entre los 5 y 12 años de edad). Adicionalmente, se ha relevado que el $99 \%$ de los jugadores/as han participado en los juegos deportivos de la secundaria, mostrando una altísima correlación con la continuidad competitiva en el deporte.

\footnotetext{
"Visión de Futuro" Año 18, Volumen $N^{0} 25$ N 1, Enero - Junio 2021 - Pág 227-250

URL de la Revista: http://visiondefuturo.fce.unam.edu.ar/index.php/visiondefuturo/index

URL del Documento: https://visiondefuturo.fce.unam.edu.ar/index.php/visiondefuturo/issue/view/19

ISSN 1668 - 8708 - Versión en Línea

E-mail: revistacientifica@fce.unam.edu.ar
} 
Claramente los hábitos deportivos incorporados a lo largo de la infancia y especialmente en la adolescencia determinan la continuidad de la práctica en la juventud. La adolescencia se configura como una etapa clave en el proceso de desarrollo de un individuo; este tratamiento específico recibe una atención especializada en el ámbito médico, psicológico, social, cultural, educativo y también económico (como consumidores).

Tabla №8: Hábitos deportivos

\begin{tabular}{|c|c|c|}
\hline Edad & \multicolumn{2}{|c|}{ Jugadores } \\
\hline 05 a 12 años & 222 & $44 \%$ \\
\hline 13 a 17 años & 334 & $67 \%$ \\
\hline 18 a 25 años & 253 & $50 \%$ \\
\hline más de 26 años & 49 & $10 \%$ \\
\hline Total & 501 & \\
\hline
\end{tabular}

Fuente: Elaboración propia

En este sentido, se observa que la práctica deportiva es transversal a todas estas dimensiones y de suma importancia en el desarrollo integral del individuo. En relación a la proyección deportiva de los jugadores/as, fue posible observar que el $49 \%$ de los participantes de los Juegos están federados.

\section{B.4.2 Análisis de la práctica deportiva con perspectiva de género}

Otro indicador interesante, surge al evaluar la participación de hombres y mujeres en el deporte. De acuerdo a los datos obtenidos a participación femenina (42\%) todavía no alcanza la paridad. Esta parte del análisis es importante para la definición de incentivos a la participación femenina en el deporte universitario.

La perspectiva de género permite ampliar el análisis de los juegos y considerando que el deporte es uno de los ámbitos donde más se ha hecho por la igualdad de género, los resultados parecen alentadores.

Tal como puede observarse en la Tabla №9, es notable que la proporción de varones supera a las mujeres por pocos puntos en todos los rangos de edad, pero llama la atención en el rango 24-26 años donde los varones llegan al 73\% de participación en relación al $27 \%$ de mujeres.

\footnotetext{
"Visión de Futuro" Año 18, Volumen N $^{0} 5$ N 1, Enero - Junio 2021 - Pág 227-250

URL de la Revista: http://visiondefuturo.fce.unam.edu.ar/index.php/visiondefuturo/index

URL del Documento: https://visiondefuturo.fce.unam.edu.ar/index.php/visiondefuturo/issue/view/19

ISSN 1668 - 8708 - Versión en Línea

E-mail: revistacientifica@fce.unam.edu.ar
} 
Tabla №9: Perspectiva de género - Rango de edad de los/las participantes

\begin{tabular}{|c|c|c|c|c|c|}
\hline Edad & \multicolumn{2}{|c|}{ Mujeres } & \multicolumn{2}{c|}{ Varones } & Totales \\
\hline 18 a 20 años & 66 & $48 \%$ & 71 & $52 \%$ & 137 \\
\hline 21 a 23 años & 81 & $43 \%$ & 107 & $57 \%$ & 188 \\
\hline 24 a 26 años & 27 & $27 \%$ & 73 & $73 \%$ & 100 \\
\hline 27 años o más & 38 & $50 \%$ & 38 & $50 \%$ & 76 \\
\hline Total & $\mathbf{2 1 2}$ & & $\mathbf{2 8 9}$ & & \\
\hline
\end{tabular}

Fuente: Elaboración propia

En tanto en la Tabla №10 se presenta la información relativa a los hábitos deportivos, es decir a la práctica de deporte a lo largo de la infancia, adolescencia y juventud. En este caso, la participación de varones supera en todos los rangos etarios a la mujer.

Tabla №10: Perspectiva de género - Hábitos deportivos de los/las participantes

\begin{tabular}{|c|c|c|c|c|c|}
\hline Edad & \multicolumn{2}{|c|}{ Mujeres } & \multicolumn{2}{c|}{ Varones } & Totales \\
\hline 05 a 12 años & 85 & $38 \%$ & 137 & $62 \%$ & 222 \\
\hline 13 a 17 años & 135 & $40 \%$ & 199 & $60 \%$ & 334 \\
\hline 18 a 25 años & 93 & $37 \%$ & 160 & $63 \%$ & 253 \\
\hline más de 26 años & 17 & $35 \%$ & 32 & $65 \%$ & 49 \\
\hline Total & 212 & & 289 & & \\
\hline
\end{tabular}

Fuente: Elaboración propia

\section{CONCLUSIÓN}

En la actualidad, las organizaciones deportivas, entiéndase ministerios o secretarías de deporte, clubes, asociaciones, federaciones, son las encargadas de gestionar la actividad deportiva y su función se concentra en el desarrollo del deporte, la creación o mejoras de la infraestructura, la gestión de recursos económicos y humanos y la organización de eventos entre otras actividades. Estas organizaciones, pasan por procesos de toma de decisiones en las cuales deben asignar prioridades y para hacerlo de manera racional necesitan contar con herramientas adecuadas para la gestión de la información. El análisis de impacto mediante el uso de multiplicadores representa una herramienta significativa para la toma de decisiones a la hora de impulsar determinados sectores productivos y poder medir el efecto del shock total en la economía. (Beyrne, 2015)

Inspirado en ello, el presente estudio ofrece dos herramientas: por un lado, el uso de los multiplicadores de la matriz Insumo-Producto y por otro encuestas para relevar información de calidad, que le permitirán a las organizaciones deportivas evaluar el impacto económico y el legado de los eventos deportivos que organicen. Con indicadores concretos que ponen de manifiesto la faceta económica y social del deporte, como la repercusión mediática,

\footnotetext{
"Visión de Futuro" Año 18, Volumen $N^{0} 25$ N 1, Enero - Junio 2021 - Pág 227-250

URL de la Revista: http://visiondefuturo.fce.unam.edu.ar/index.php/visiondefuturo/index

URL del Documento: https://visiondefuturo.fce.unam.edu.ar/index.php/visiondefuturo/issue/view/19

ISSN 1668 - 8708 - Versión en Línea

E-mail: revistacientifica@fce.unam.edu.ar
} 
generación de capital humano, capital social, hábitos deportivos y otros que normalmente no se visualizan.

En concreto, el modelo de análisis de impacto consta una serie de pasos diferenciados que se presentan de manera secuencial y que partiendo del efecto directo e indirecto permite obtener el impacto económico total para al área territorial de referencia que se plantee en el estudio. Hay que destacar que esta herramienta se ha desarrollado con el objetivo de facilitar y mejorar la realización de este tipo de estudios en el ámbito deportivo, pero también es aplicable a eventos culturales, educativos, recreativos, etc que atraigan visitantes.

Sin embargo, no se pueden dejar de mencionar dos aspectos que pueden generar controversia en las aplicaciones del modelo y que se relacionan con los supuestos subyacentes al modelo input-output por una parte y a la antigüedad de la Matriz InsumoProducto de Argentina, por otra.

K. Porter y Fletcher (2008) presentan una serie de críticas a la utilización del modelo input output para medir impactos de largo plazo en la economía, en relación con la función de producción y las funciones de ofertas de insumos. Sobre los supuestos que subyacen al modelo no es posible realizar ajustes que subsanen esta debilidad.

Así mismo señalan que las estimaciones son elaboradas a partir de encuestas a los asistentes o proyecciones de eventos pasados que tienden a sobreestimar el gasto de los visitantes. En el estudio de impacto de los Juegos, el 86,16\% de las encuestas fueron respondidas por visitantes, por lo que se considera robusto el dato de gastos nuevos atribuibles al evento.

El segundo aspecto a considerar es la utilización de una matriz con 15 años antigüedad, que podría no estar reflejando adecuadamente las relaciones intersectoriales actuales. En este sentido Porta F. (20016) analiza que el régimen de crecimiento predominante desde 2003, que ha favorecido una evolución muy positiva en la mayoría de los indicadores socioeconómicos y en la calidad de vida de la población, no se apoyó en un cambio estructural significativo. En líneas generales la composición del PBI no ha variado significativamente en relación con los años 90 . A su vez analizando cada uno esos sectores tampoco se han producido cambios significativos en el tipo, la gama y la variedad de los productos o servicios producidos.

Salvando estas apreciaciones, el necesario proceso de recapitulación permite extraer una serie de conclusiones referidas a la celebración de eventos deportivos y la cuantificación y medición del impacto económico generado por los mismos. En términos del estudio realizado, se verifica que los Juegos han dejado un legado en capital humano valioso en cuanto a la formación de estudiantes de la salud, el diseño, turismo y comunicación. Cómo 
así también el equipo organizador sigue desarrollando habilidades para la planificación y ejecución de eventos deportivos (know how), fortaleciendo lasos con organismos estatales necesarios para la realización de futuros eventos deportivos en la provincia de misiones.

Se manifestó la necesidad de planificar fixture, que permita la visita de lugares de interés turísticos que posee la provincia y que permitirían esperar un mayor efecto económico a corto y largo plazo. Este evento deportivo, también impactó de forma favorable en las arcas de los estados provinciales y nacionales generando ingresos adicionales por los consumos tanto de organizadores como de visitantes.

Como conclusión los casi $\$ 4.000 .000$ aplicados a la organización de los Juegos se traducen en más de $\$ 12.000 .000$ en movimiento económico a partir de la generación de consumo de los visitantes, de los proveedores del evento (efectos directos) y de los proveedores de insumos para esos proveedores (efectos indirectos). Es decir que, por cada peso aplicado a la realización del evento, la economía recibe un impulso en su demanda que se multiplica 3 veces en ingresos para toda la cadena de valor que se genera a partir de esa demanda inicial.

Por último, este estudio sirve de base a futuras investigaciones sobre los factores que contribuyen a la práctica deportiva de la comunidad, los análisis con perspectiva de género, el desarrollo de capital humano y social, turismo deportivo entre otros. Todos estos temas relacionados directamente con el concepto de impacto y legado, buscando identificar la incidencia del deporte sobre aspectos de relevancia económica y social, a fin de diseñar y evaluar estrategias tanto públicas como privadas que permiten mejorar la tasa de participación deportiva en nuestra sociedad.

\section{REFERENCIAS}

Betrán, J. (2005). Adolescencia, deporte y crecimiento personal. Apuntes para el siglo XXI (Editorial).

Brunet, F. (2016) Análisis del impacto económico de los juegos olímpicos. Mosaico olímpico, investigación multidisciplinar y difusión de los estudios olímpicos CEO-UAB, 20 años

Beyrne, G. (2015). "Análisis de Encadenamientos Productivos y Multiplicadores a partir de la construcción de la Matriz de Insumo - Producto Argentina 2004". Documento de Trabajo N¹3. Secretaría de política económica y planificación del desarrollo".

Coalter, F. (2007) Sports Club, Social capital and social regeneration: "ill-definied interventions with hard to follow outcomes"? Sport in society 10, 4 537-559.

Comité Olímpico Argentino (2014). Manual de Administración Deportiva

\footnotetext{
“Visión de Futuro" Año 18, Volumen $N^{0} 25$ N 1, Enero - Junio 2021 - Pág 227-250

URL de la Revista: http://visiondefuturo.fce.unam.edu.ar/index.php/visiondefuturo/index

URL del Documento: https://visiondefuturo.fce.unam.edu.ar/index.php/visiondefuturo/issue/view/19

ISSN 1668 - 8708 - Versión en Línea

E-mail: revistacientifica@fce.unam.edu.ar
} 
Cortés Fregoso, J. y Sepúlveda Nuñez, M. (2006). "Medición del Impacto Económico del Deporte". X Congreso Anual de la Academia de Ciencias Administrativas. Universidad de Guadalajara, México.

Crompton, J. (2006). Economic Impact studies: Instruments for political Shenanigans? Journal of Travel Research, 45: 67-82.

Hughes, K.; Kirk, D. y Long, J (2010) El Legado Social de los macro-eventos deportivos y los juegos olímpicos y paralímpicos de Londres 2012. Citus, Altius, Fortius: Humanismo, sociedad y deporte. Investigaciones y ensayos 3 (1), pp 19-38

Instituto Nacional de Estadísticas y Censo. "Comprendiendo la Matriz Insumo Producto". Documentos Metodológicos.

Kidd, B. (2003) The global sporting legacy of the Olympic movement. Paper presented at the Legacy of the Olympic Games 1984-2000, International Symposium

Lieber, S. R., Alton, D. J. y Fesenmaier, D. R. (1983). "Visitor expenditures and the economic impact of public recreation facilities in Illinois. Recreation planning and management, 36 $-54$.

López Roldán, P. y Fachelli, S. (2015). "Metodología de la Investigación Social Cuantitativa". Universidad Autónoma de Barcelona. Barcelona, España.

Medina, F. y Sánchez, R. (2005) Actividad físico-deportiva, turismo y desarrollo local en España. PASOS Revista de turismo y patrimonio cultural. Vol. 3 №1 págs. 97-107. 2005 [https://doi.org/10.25145/j.pasos.2005.03.006]

Misener, L y Mason, M. (2006) Creating community networks: Can sporting event offer meaningful sources of social capital? Managing Leisure 11:1, 39-56

Potter, K. H. (2009) The development of a plan to maximise the benefits of the staging of the 2012 Olympic and Paralympic Games in London. Report prepared for Leeds Metropolitan University. Unpublished.

Porta, F. (2016) La estructura productiva argentina. Problemas vigentes y desafíos pendientes. Voces en el Fenix, 53: 90-97.

Porter, F. K. y Fletcher, D. (2008). The Economic Impact of the Olympic Games: Ex Ante Predictions and Ex Poste Reality.

Preuss, H. (2006). Lasting effects of major sporting events [www.idrottsforum.org / ISSN16527224] 2006-12-13

Preuss, H. (2007) The conceptualization and measurement of mega sport tourism. Journal of Sport \& Tourism, 12 (3-4), 207-227

\footnotetext{
"Visión de Futuro" Año 18, Volumen $\mathrm{N}^{0} 25 \mathrm{~N}^{0}$ 1, Enero - Junio 2021 - Pág 227-250

URL de la Revista: http://visiondefuturo.fce.unam.edu.ar/index.php/visiondefuturo/index

URL del Documento: https://visiondefuturo.fce.unam.edu.ar/index.php/visiondefuturo/issue/view/19

ISSN 1668 - 8708 - Versión en Línea

E-mail: revistacientifica@fce.unam.edu.ar
} 
Preuss, H. (2010). Economic dimension of the Olympic Games. University lecture on the Olympics [online article]. Barcelona: Centre d'Estudis Olympics (UAB). International Chair Olympism (IOC-UAB)

Salgado-Barandela, J.; Barajas, A. y Sánchez-Fernández, P. (2017). Impacto económico del deporte: tema de interés creciente en la literatura científica

Sánchez Fernández, P. y Barajas Alonso, Angel (2012) Los eventos deportivos como generadores de impacto económico: factores clave y medición. Universidad de Vigo

Thomson, A.; Schlenker, K. y Schulenkorf, N. (2013) Conceptualizing sport event legacy. Event management, Vol 17, pp 111-122. E-ISSN 1943-4308

Walters, G. (2013) Gestionando el legado de un evento deportivo: el caso de los Juegos Olímpicos de Londres 2012. Birkbeck. University of London.

Weed, M; Coren, E y Fiore, J. (2009) A systematic review of the evidence base for developing a phisical activity and heatl legacy from the London 2012 olympic and paralympic games, Cantebury, SPEAR.

\section{RESUMEN BIBLIOGRÁFICO}

\section{Eva Muguerza}

Licenciada en Economía por la Universidad del Salvador, Doctorando en Economía en la Universidad Católica Argentina. Docente de economía y estadística, investigadora y extensionista en la Universidad Nacional de Misiones. Coordinadora del Observatorio Económico y Social del Deporte y la Actividad Física. Campo de interés: economía del deporte.

\section{Natalia Ojeda}

Licenciada en Economía por la Universidad Nacional de Misiones. Actualmente analista de datos y economista en el Instituto Provincial de Estadística y Censos). Docente e investigadora de la UNaM en el ámbito de la economía social y la competitividad regional.

\section{Tania Elizabet Ruff}

Licenciada en Economía (UNaM). Se desempeña como JTP en la Cátedra de Políticas Económicas y el Rol del Estado (Lic. En Trabajo Social - FHyCS - UNAM) y colabora en el Observatorio Económico y Social del Deporte y Actividad Física - UNaM.

\section{Francisco Mauricio Rosenfeld y Sommer}

Magister en Administración Estratégica de los Negocios por la (UNaM 2019). Auxiliar de docencia de economía, comercialización, investigador y extensionista en la UNaM. Miembro del Observatorio Económico y Social del Deporte y la Actividad Física. Campo de interés: economía del deporte y Territorialidades Transfronterizas.

\footnotetext{
"Visión de Futuro" Año 18, Volumen N $^{0} 5$ N 1, Enero - Junio 2021 - Pág 227-250

URL de la Revista: http://visiondefuturo.fce.unam.edu.ar/index.php/visiondefuturo/index

URL del Documento: https://visiondefuturo.fce.unam.edu.ar/index.php/visiondefuturo/issue/view/19

ISSN 1668 - 8708 - Versión en Línea

E-mail: revistacientifica@fce.unam.edu.ar
} 